\title{
It's All About Contrast: Multifrequency Resonant and IR Methods in AFM
}

Greg Haugstad ${ }^{1}$ and Andrew Avery ${ }^{2}$

${ }^{1}$ University of Minnesota, Characterization Facility, Minneapolis, Minnesota, United States, ${ }^{2}$ Unilever Research, Bebington, England, United Kingdom

Nanoscale materials differentiation via an atomic force microscope (AFM) has been a "holy grail".[1] Since AFM's began to appear in core facilities in academia circa 1990, however, facility managers have been perplexed by the "race to the bottom" to primarily use these sophisticated tools for topographic imaging (and "version 0.9" metrics thereof such as $Z_{\mathrm{rms}}$ ). Happily there have been a sufficient number of "freewheeling" users - perhaps with less straightjacketed research budgets (and silenced smartphones) to reward the heroic efforts of AFM vendors in developing transformative methods for generating materials contrast. Here we exemplify some of the more pioneering (now commercial) methods.

In the first part of this presentation we discuss a resonant, multifrequency method developed by Intermodulation Products AB (Stockholm) - vibrating the microcantilever (to which the tip is attached) at two tones near the fundamental flexural resonance - to generate dozens of tones of response due to nonlinear tip-sample interaction with distance (a well-known mathematical concept in electrical engineering termed intermodulation). This method both expands contrast mechanisms (images of amplitude and phase at each of 40 mixing tones, separated by integer multiples of the driving-tone difference frequency) and reconstructs the distance dependence of conservative and dissipative material response at each image pixel via a 40-term discrete Fourier transform of tip motion ( $\mathrm{Z}$ position and velocity). On surfactant-based films pertinent to lubrication and superhydrophilicity (i.e., our collaborations in the medical device and cleaning industries), these methods are used to sense both the elastic and viscous response to the AFM tip. Machine learning (linear discriminant analysis) is further applied to cluster-analyze distance-dependent elastic and dissipative force fingerprints, and thereby improve signal/noise, whereby more sharply differentiated film domains. Figure 1 exemplifies the case of a submonolayer, two-surfactant lubrication film on human hair.

In the second part of this presentation we discuss "AFM-IR" imaging (using a Bruker NanoIR3, Santa Barbara). The described methods sense pulsed infrared radiation absorption, and thereby chemical bonding, at the scale of the tip-sample interface $(\sim 10 \mathrm{~nm})$. The first sub-method is "bimodal" - driving the AFM microcantilever at its fundamental resonance for the purpose of tracking topography while generating "phase" images (i.e., conventional "tapping mode") - and pulsing IR laser light at the tipsample interface at a frequency that, when added to the fundamental, produces excitation at the microcantilever's higher eigenfrequency if the material absorbs at the chosen IR-laser wave number. This sub-method is necessary for samples that cannot withstand the shear forces at a sliding AFM tip under continuous contact (i.e., soft or weakly bound). The method does not, however, provide the strongest spectral signal/noise. The second sub-method, which indeed is much higher signal/noise, presses the AFM tip continuously against a surface (i.e., in sliding contact, enabling friction force imaging as a side benefit) while pulsing the IR laser at the fundamental contact resonance frequency. This frequency can vary, however, quite substantially from one contact point to another because of material differences (e.g., modulus) or contact geometry (i.e., valley vs. hilltop). Thus a phase-locked loop is invoked so as to offset shifts in the contact resonance frequency and thereby better control the measurement; and, as a by-product, provide images of this shifting frequency and thereby variable contact stiffness from location to location. Hence the information content in this "contact resonance" sub-method can be large... which is extremely 
exciting to the "freewheeling" user! Figure 2 exemplifies the case of a poly n-alkyl methacrylate polymer blend that generates no contrast in conventional AFM property-sensitive modes (e.g., phase, friction, adhesion) at room temperature. Here the simple difference in side-chain provides ample IR absorption contrast. It should be noted, however, that this chemical probe extends of order $100 \mathrm{~nm}$ into the material, a concept that is foreign to most AFM users. Thus one must become acclimated to viewing both "surface" images and deeper images, though simultaneously acquired.

\section{LDA component 0}

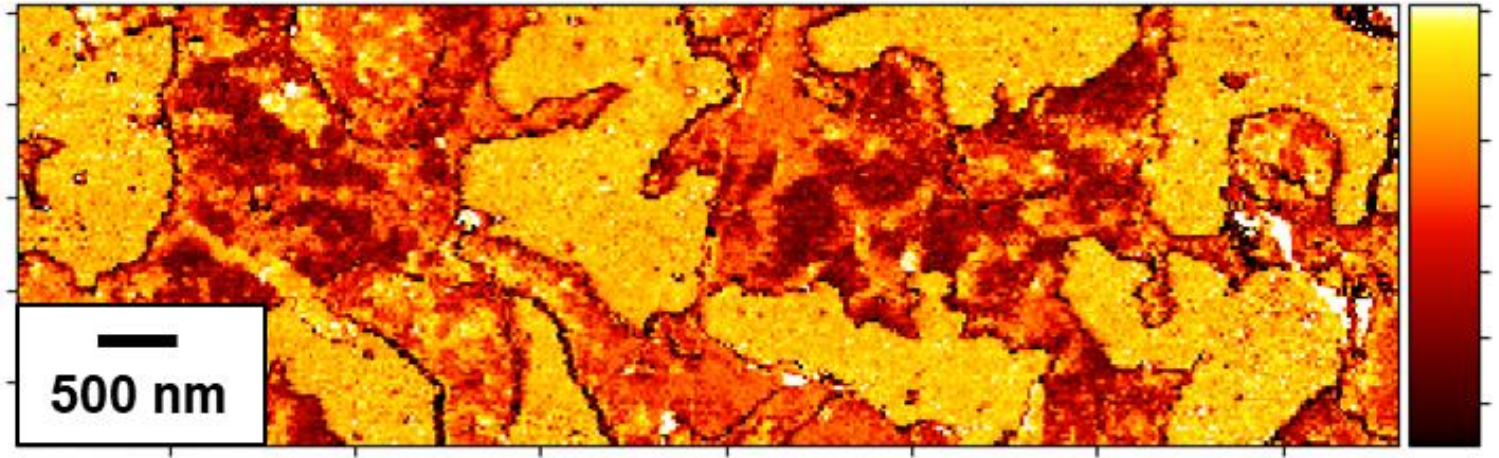

Figure 1. Linear discriminant analysis (LDA) cluster (of distance dependence) rendering of contrast between two liquid-phase surfactant domains (darker, orange/red) and bare hair as substrate (bright, yellow).

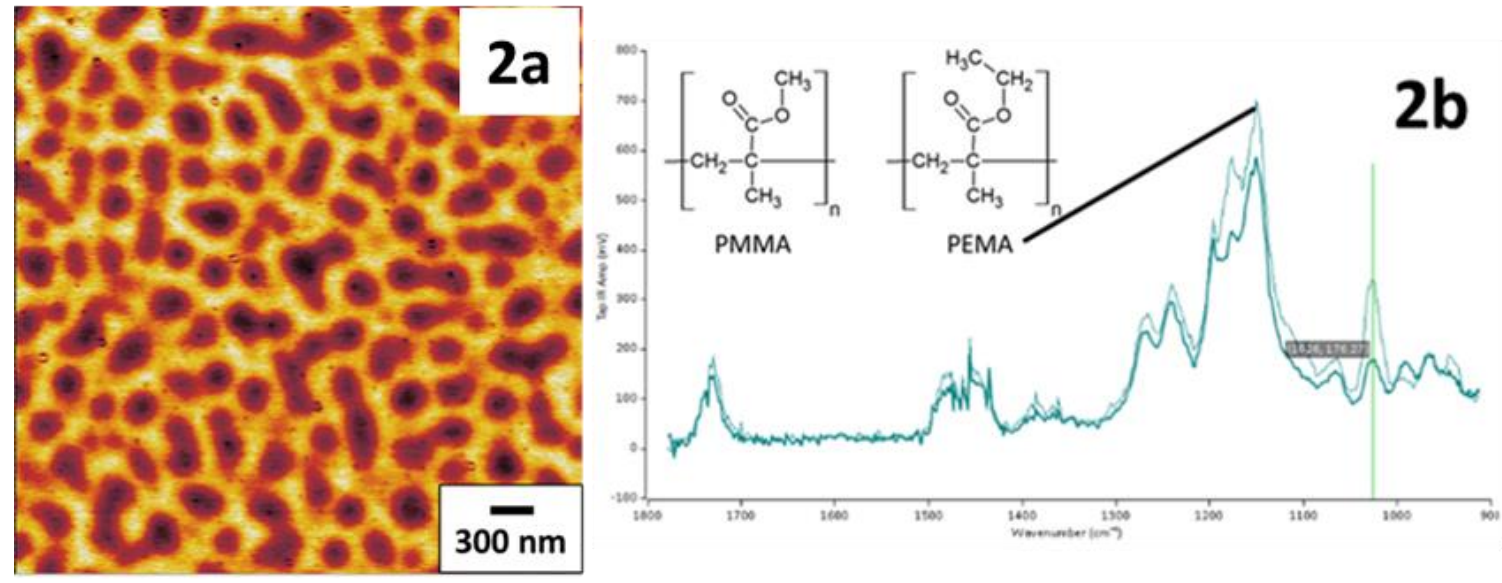

Figure 2. (a) IR absorption (brighter being greater) at a wave number of $1026 \mathrm{~cm}-1$ on a 50:50 PEMAPMMA blend; (b) IR absorption spectra (1780-910 cm-1) collected at two characteristic locations on PEMA (thin plot) and PMMA (thick plot). Green vertical line denotes the wave number at which the pulsed laser produced the absorption image of $2 \mathrm{a}$ (PEMA being brighter).

References

[1] G. Haugstad, Atomic Force Microscopy: Understanding Basic Modes and Advanced Appplications (Wiley, 2012). 